\title{
LA FROMAGERIE EN CONTINU (1)
}

par

\author{
M. Pierre-STENNE
}

A la base de la plupart des procédés de fabrication de fromage en continu, se trouve une découverte amorcée par Effront aux U.S.A. en 1917 et précisée par Berridge en Grande-Bretagne en 1942, concernant l'action de la présure sur le lait. Ces chercheurs; ont en effet établi que la présure avait une action enzymatique et une action coagulante qui pouvaient être facilement dissociées: Ils ont mis en évidence que l'action enzymatique se développe convenablement à basse température à un rythme fonction de la température, mais suffisamment lent pour qu'une coagulation à cette température n'intervienne qu'au bout d'un temps relativement long, soit plusieurs jours. Par contre, l'action de coagulation peut être obtenue sur un tel lait emprésuré à froid, en quelques secondes, lorsque ce lait est porté à une température de l'ordre de $30-35^{\circ} \mathrm{C}$.

Cette découverte est à la base de la plupart des procédés de fabrication de caillé de fromagerie en continu qui ont été développés récemment ou sont en cours de développement actuellement.

En dehors du procédé français dont nous allons parler, nous citerons les 2 plus connuś, c'est-à-dire, le procédé anglais Berridge et le procédé hollandais du Nizo.

De façon schématique le premier procédé (breveté) consiste à emprésurer le lait normal, convenablement standardisé, préalablement pasteurisé et ensemencé en ferments à une dose normale, à une température de l'ordre de $4^{\circ} \mathrm{C}$., au moyen d'une quantité de présure analogue ou légèrement supérieure à Ja dose normale. Le lait froid ainsi emprésuré est stocké à froid pendant plusieurs heures, nécessaires poür que l'action enzymatique de la présure soit complète.

A l'issue de cette période de stockage, le lait emprésuré est réchauffé en le faisant couler sur la surface d'un tambour rotatif, genre Hatmaker, dont la paroi est chauffée par circulation interne d'eau chaude ou de vapeur. Le lait caille immédiatement ou très rapidement sous forme d'une pellicule qui est ensuite coupée et détachée par un couteau racleur. Le caillé est alors repris dans un système de transport où s'effectue la synérèse, la séparation du sérum et du caillé, et éventuellement le lavage, la cuisson, etc.

(1) La Technique Laitière, 1964, 463, 13. 
La nécessité d'obtenir de façon pratiquement instantanée un caillé suffisamment ferme pour être travaillé sans pertes excessives, conduit à chauffer le lait sur le cylindre à une température anormalement élevée. Il en résulte d'une part, un " coiffage " du grain et d'autre part, une destruction presque totale des ferments lactiques.

Ces conditions, qui risquent d'aboutir simplement à la fabrication de caséine et non pas de fromages, réduisent sensiblement l'intérêt du procédé. Néanmoins certains facteurs de correction ont été étudiés, en particulier, l'augmentation de la dose de présure, ou l'addition de ferments après caillage, mais sans résultats appréciables semble-t-il.

Le deuxième procédé (également breveté), mis au point en 1962 par le Nizo en Hollande, part du même principe d'emprésurage à froid d'un lait normal convenablement standardisé, pasteurisé et ensemencé en ferments lactiques dans des conditions classiques. Pour pallier les inconvénients du procédé précédent, le chauffage du lait emprésuré est réalisé dans le procédé Nizo de façon très douce au moyen d'un échangeur de ohaleur à plaques spécial. Ce chauffage est très délicat, car il faut à tout prix éviter qu'un caillage du lait ne se produise dans l'appareil. Non seulement une régulation de température absolument parfaite s'impose, mais également l'emploi d'un fluide chauffant (eau chaude) à une température voisine de la température de chauffage du lait. Ces impératifs conduisent à une surface d'échange importante donc un temps de séjour relativement long (20 secondes environ) du lait dans le réchauffeur. Le lait ainsi réchauffé, à une température inférieure, mais voisine de la température de coagulation, passe alors dans un appareil vertical et cylindrique appelé coagulateur, dans lequel le caillé se forme progressivement, poussé par le lait qui circule de bas en haut. A la partie supérieure, le caillé est découpé par un système spécial et ensuite introduit dans un tambour rotatif horizontal pour y subir les opérations de synérèse et de séparation de sérum et éventuellement de lavage.

L'opération de fabrication du caillé dure environ 25 minutes.

Ce système employé jusqu'à présent au stade pilote pour fromage type $E d a m$ permet d'obtenir semble-t-il des fromages ayant des normes et caractéristiques acceptables.

Parmi les inconvénients du procédé, on peut citer le temps très long d'emprésurage à froid, la difficulté de faire un caillé ayant une fermeté convenable, les pertes et le rendement faible, le temps de fabrication et le manque de souplesse du système.

En dehors de ces deux procédés nous citerons le procédé Le Bœuf "Fromatic ", qui avait été exposé il y a 5 ans au Salon de l'Equipement Laitier à Paris et qui est la mécanisation d'opérations classiques de fromagerie, le lait étant emprésuré et coagulé en 
augets se déplaçant lentement et uniformément sur une chaîne, avec poste de tranchage et de moulage automatique. Ce système, dont l'encombrement est considérable et qui certainement manque de souplesse est toujours à l'état de prototype.

Citons également un procédé russe, consistant en un grand cylindre rotatif dans lequel s'effectuent en continu les opśrations d'emprésurage, de caillage, tranchage, synérèse et évacuation du sérum. Il semble que ce procédé soit également resté au stade de prototype et n'ait pas eu de développement sensible.

\section{Ce que sont les procédés Hutin (1)}

Les procédés Hutin (brevetés ou brevets déposés dans le monde entier) s'apparentent dans une certaine mesure avec les procédés Berridge et Nizo, en ce sens qu'ils font appel également à l'emprésurage à froid du lait. Ils diffèrent cependant de tous les procédés connus jusqu'à ce jour - et ceci est d'une importance primordiale, à de très nombreux points de vue - par le fait que la matière première utilisée est non plus du lait normal, mais du lait concentré, préalablement standardisé et pasteurisé.

Les Services de Recherches des Etablissements Hutin à l'origine sont partis de l'idée, jugée peut-être comme simpliste aujourd'hui, que si l'on retirait une partie de l'eau du lait avant fabrication, celle-ci s'en trouverait simplifiée, les volumes à traiter étant alors plus réduits et l'égouttage à priori facilité.

L'idée d'employer du lait concentré comme matière première de base en fromagerie était par ailleurs séduisante ear, une telle technique, en dehors de ces considérations économiques de fabrication, donnait à priori, la possibilité de réaliser par concentration une matière première homogène de "grand mélange ", sous un faible volume, ce qui dans le cas des pâtes molles et d'une façon générale des fromages vendus à la pièce ouvrait la voie à la fabrication de fromages à poids égaux.

Après de longues et patientes recherches, orientées à l'origine dans le domaine des pâtes molles, une technique dite " procédé $\mathrm{SH}$ 11 ) fut mise au point en 1962.

Cette technique, appliquée actuellement à l'échelle industrielle, repose sur une modification de la structure physico-chimique du lait au cours de la concentration. Il a été mis en évidence, en effet, que le rendement en fromagerie est fonction de la dimension des particules de phosphocaséinate du lait. Ce phénomène est d'ailleurs connu pour le lait normal et l'on sait depuis un certain temps que c'est la différence de taille des particules de phosphocaséinate qui caractérise les laits lents des laits rapides.

(1) Inventeur : Pierre Stenne. 


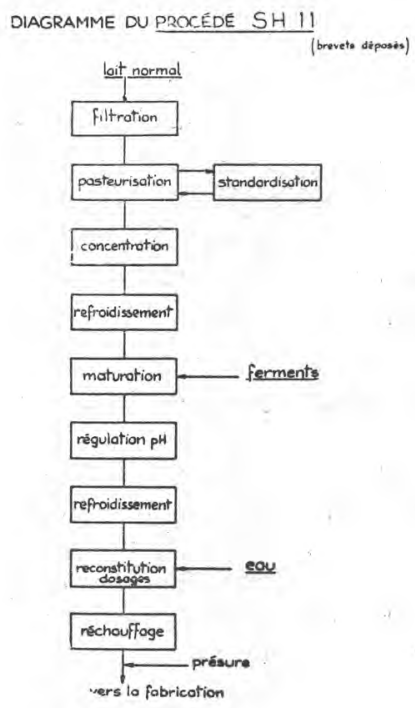

Fig. I

Lors de la concentration, ce phénomène est considérablement accentué, après concentration on obtient des agrégats de taille 50 à 100 fois celle de la particule initiale de phosphocaséinate.

Ces agrégats sont "consolidés " par un stockage, mais par contre sont progressivement "dilués " ou "dissous " lorsque l'on revient à l'état de concentration initial.

- La présence dans le lait de particules de phosphocaséinate sous cette forme d'agrégat a pour conséquence, lors du caillage du lait, la formation d'une structure rectifiée, qui constitue une sorte de filtre, retenant en particulier aussi bien de la matière grasse que de très fines particules de caséine et provoquant par là, une augmentation de rendement substantielle.

Ce point à lui seul constituait au départ un aspect très positif de procédé SH 11 mais encore, convenait-il de tirer parti de ]'intérêt du phénomène, tout en fabriquarit des fromages de composition, d'aspect et de goût analogues ou supérieurs à ceux des fromages équivalents fabriqués selon les méthodes classiques.

Les travaux ont alors porté d'une part sur la recherche des conditions optimum de concentration, avec en particulier obtention d'un produit convenablement pasteurisé, mais non dégradé par la chaleur, et d'autre part, sur la richesse optimum en matières sèches du lait à mettre ensuite en œuvre pour la fabrication selon les procédés classiques. 
Il a été ainsi vérifié qu'une concentration trop forte (45 p. 100 EST) (1) ne permettait pas le développement d'une flore lactique; et que d'autre part un affinage convenable des fromages ne pouvait se réaliser que lorsque le rapport lactose/protéines était faible.

Comme le lactose est extrait du caillé par l'eau lors de l'égouttage, un appauvrissement convenable en lactose ne pouvait donc se faire qu'en maintenant dans le lait une teneur en eau suffisante.

Partant de ces principes, le procédé SH 11 qui a alors été mis au point, consiste à concentrer le lait normal, convenablement standardisé et pasteurisé jusqu'à un taux optimum d'EST et refroidir le concentré obtenu après l'avoir ensemencé en ferments lactiques pour réaliser une protection $\mathrm{du}$ milieu et une acidification. convenable. Ceci constitue la phase de grossissement des molécules. Après quelques heures de stockage le lait est dilué au moyen d'eau, la dilution étant réalisée au moyen d'un système de mesure très simple et précis, à un taux d'EST défini, voisin du taux d'EST du lait normal ou légèrement supérieur. Le lait ainsi reconstitué est ensuite immédiatement réchauffé dans un échangeur à plaques, à la température classique d'emprésurage, emprésuré et envoyé normalement et classiquement en fabrication.

Ce procédé qui est l'amorce des procédés SH 12 et SH 13 de fabrication en continu que nous décrirons plus loin, présente déjà en lui-même nombre d'aspects positifs.

\section{a) Augmentation du rendement maigre.}

La structure rectifiée du caillé permet de retenir des poussières, de caséine entraînées par le sérum dans les procédés classiquès.

Des études précises, portant sur des fabrications de laboratoires avec bilans précis, sur des fabrications semi-industrielles et industrielleś avec des relevés statistiques établis sur plusieurs mois de travail industriel avec fabrications classiques de comparaison (toutes choses égales par ailleurs), ont mis en évidence des augmentations de rendement maigre comprises entre 7 et 12 p. 100 selon: Jes types de fromages.

Ces travaux de contrôle ont d'ailleurs été réalisés avec la colla-boration et sous le contrôle de M. le Professeur Camus, Directeur à la Recherche Agronomique à Paris et l'étude qui en est résultée a fait l'objet d'une publication récente.

L'intérêt économique de cette augmentation de rendement maigre est considérable, compte tenu non seulement de la valorisation de la matière sèche dégagée, mais également des frais d'exploitation et d'investissement du matériel de concentration mis en cuvre et de la diminution de production de sérum rapportée en matières sèches totales.

(1) EST = Extrait Sec Total. 
Il convient de préciser que l'augmentation de rendement constatée, n'est pas dûe à un excès d'eau dans la pâte, pas plus d'ailleurs qu'à une retenue plus importante dans le fromage de substances indésirables telles que lactose et albumine; il s'agit par ailleurs, bien entendu de mesures se rapportant à des fromages de même humidité et même matière grasse, obtenus à partir d'un lait identique au départ, c'est-à-dire, de même composition et ayant été pasteurisés aux mêmes températures.

\section{b) Réduction des pertes de matières grasses.}

La concentration du lait entier, standardisé provoque une répartition homogène de la matière grasse dans le lait concentré.

D'autre part cette répartition est stable durant le stockage en raison de la viscosité du concentré.

Enfin le réseau des particules de phosphocaséinate retient mieux et plus complètement la matière grasse.

Ces phénomènes aboutissent à pertes de matière grasse réduites d'environ $1 \mathrm{gr} / \mathrm{kg}$ par rapport à celles du sérum normal pour les pâtes molles par exemple. Cette diminution des pertes peut être encore beaucoup plus sensible dans le cas d'autres types de fromages. Ainsi au cours d'essais semi-industriels portant sur la fabrication de fromages type Cheshire les pertes ont été ramenées de 6,2 gr/ kg pour les procédés classiques à $3,3 \mathrm{gr} / \mathrm{kg}$ de sérum.

\section{c) Qualité des fromages.}

De nombreux échantillons de fromages provenant de fabrications semi-industrielles selon le procédé SH 11 ont été comparés par des experts avec des produits fabriqués à partir d'un même lait selon les méthodes classiques.

Ces échantillons étaient relatifs à une grande variété de types de fromages et les dégustations opérées selon les règles les plus strictes, ont mis en évidence la supériorité de goût, de texture et de tenue des fromages fabriqués selon le procédé SH 11.

Cette amélioration des qualités physiques et organoleptiques s'explique en partie par le fait que la maturation du lait est réalisée sur un produit concentré et est favorisée par la concentration du milieu. D'autre part, l'acidité du concentré inhibe le développement des bactériophages ou d'autres bactéries nuisibles telles que cryophiles, etc. Enfin, lors de la concentration le lait subit un véritable dégazage qui améliore ses qualités organoleptiques.

La médaille d'or du Concours Général Agricole 1964 attribuée aux Etablissements J. Hutin pour les fromages à pâtes molles, type Carré de l'Est, correspondait d'ailleurs à des fromages fabriqués selon ce procédé. 


\section{d) Constance des fromages.}

L'une des préoccupations essentielles des fabricants de fromages est la constance des produits fabriqués. Cette constance doit s'appliquer aussi bien aux qualités organoleptiques, qu'à la texture, à l'aspect et au poids, surtout dans le cas des fromages vendus à la pièce. Le procédé SH 11 permet sans aucun doute une amélioration de la constance des fromages à tous les égards.

En effet, le lait étant normalement de composition variable, non seulement d'une saison ou d'un jour à l'autre, mais également d'une zone de ramassage à l'autre, le fromager se trouve dans l'obligation normalement pour pallier de telles hétérogénéités, de réaliser un stockage de très grande capacité, qui lui permettra de réaliser un lait dit de "grand mélange". Il n'est pas rare de voir aujourd'hui à cet effet des tanks de stockage de capacité de 30000 litres ou même de 50000 litres. Cependant, l'hétérogénéité susbiste d'un tank à l'autre.

Le problème est différent dans le cas du procédé SH 11 puisque toute la matière première relative à la fabrication d'une journée ou même plus peut être stockée dans un seul tank.

Le fromager a donc à sa disposition un lait de composition connue et parfaitement homogène du point de vue acidité, composition et matières grasses.

La redilution, deuxième phase du procédé $\mathrm{SH} 11$, a un taux d'EST précis voisin ou supérieur au taux d'EST du lait initial, va donc permettre des fabrications à partir d'une matière première constante.

Ceci a une répercussion sensible sur les constances de qualités organoleptiques, de la texture et du poids.

\section{e) Qualité bactériologique du lait.}

Lorsqu'un lait d'acidité par exemple $18^{\circ} \mathrm{D}$ est concentré de 12 p. 100 EST à 36 p. 100 EST, l'acidité du concentré passe à $55^{\circ} \mathrm{D}$ environ. Les ferments lactiques qui sont mis dans le concentré froid augmentent encore cette acidité. L'acidité du concentré est donc dès le début du stockage à une valeur telle que le développement de bactéries indésirables est considérablement sinon totalement inhibé. Il en est de même pour le bactériophage.

Par contre, même à la température de stockage et au taux de concentration prévus dans le procédé SH 11, les ferments lactiques se développent d'abord lentement, puis rapidement avec une augmentation très sensible de la population.

Ces effets sur la protection et l'amélioration des qualités bactériologiques du lait concentré ont été vérifiés sur des fabrications industrielles et se répercutent sur la qualité des fromages. 


\section{f) Acidité optimum du lait pour la fabrication.}

La maturation du lait concentré se traduit par un abaissement du $p \mathrm{H}$. Lorsque le $p \mathrm{H}$ optimum pour un type de fromage ou une. qualité de fromage a été déterminé, il est facile de contrôler automatiquement la maturation à l'aide d'un dispositif comprenant un $p$ Hmètre à action automatique commandant un refroidissement rapide concentré.

Cette action permet un blocage presque immédiat et définitif du $p H$ à la valeur désirée.

Ce point, à rattacher aux facteurs de constance des fromages, est à considérer également sous l'angle automaticité.

\section{g) Nouvel aspect du ramassage.}

Il est certain que le travail en lait concentré modifie de façon sensible la physionomie du ramassage, puisque le stockage du lait sous forme concentrée et son report de 12 à $24 \mathrm{~h}$ pour maturation permettent de dissocier le ramassage de la fabrication.

De même, on peut concevoir des transports de lait concèntré de centres de concentration relativement éloignés, avec réduction sensible des frais de transport et investissements peu onéreux.

\section{h) Polyvalence de l'équipement.}

Le procédé SH 11, comme d'ailleurs les procédés SH 12 et SH 13 ayant comme principe de base, la concentration du lait, l'adoption de l'un ou l'autre des procédés nécessite done l'utilisation d'un matériel de concentration spécialement conçu et installé à cet effet. Dans les installations pilotes ou industrielles réal sées jusqu'à ce jour la concentration est réalisée dans des Evaporateurs à plaques A.P.V. Ces appareils, dont les conditions de fonctionnement sont adaptées de façon précise aux normés de température et de concentration fixées pour les procédés SH 11, SH 12 et SH 13 ont été choisis en raison du faible temps de contact du produit au cours de la concentration, des conditions d'évaporation douces et uniformes propres à ce matériel, de la souplesse de marche, du faible encombrement et des possibilités d'extension de ces évaporateurs. Ils ont été et sont également étudiés pour servir alternativement à la concentration du sérum avant séchage.

Ce point a un grand intérêt en ce qui concerne l'amortissement de l'équipement.

Comme on peut le voir, le procédé SH 11 constitue en lui seul une technique séduisante en bien des points, qui apporte déjà un visage nouveau et intéressant à l'Industrie Laitière de Demain.

(A suivre) 